\title{
L'engagement des parties prenantes internes dans les démarches de Responsabilité Globale (RG) : l'intégration des indicateurs de RG dans les outils de pilotage du contrôle de gestion
}

\author{
Christophe Germain, Audencia Nantes School of Management \\ Stephen Gates, Audencia Nantes School of Management
}

\begin{abstract}
Résumé
La recherche montre que les mesures de performance liées à la responsabilité globale sont relativement peu présentes dans les outils de pilotage du contrôle de gestion. Au-delà de ce constat global, il apparaît que leur degré d'intégration croît dans les organisations de grande taille, les structures cotées en Bourse et les entreprises industrielles.
\end{abstract}

Les entreprises ont été amenées, ces dernières années, à s'interroger sur leur responsabilité vis-à-vis de l'environnement et de la société. Le concept de Responsabilité Globale (RG) qui postule de façon générale, dans la droite ligne de la " stakeholder theory " développée par Freeman (1984), que l'entreprise doit satisfaire aux exigences, besoins et intérêts de ses parties prenantes a ainsi pris de l'ampleur. Ce phénomène s'est traduit par de nombreuses publications sur le concept lui-même, l'évolution des discours et des stratégies de communication des entreprises (Martinet et Reynaud, 2001) ou le développement des dispositifs externes d'évaluation ou de notation des entreprises. En revanche, les dispositifs internes de contrôle de gestion utilisés par les entreprises pour évaluer la performance environnementale, sociale et sociétale, n'ont suscité de l'intérêt que plus récemment (Mocquet, 2005 ; Bollecker et al. 2006 ; Marquet-Pondeville et al. 2007 ; Meyssonier et Rasolofo Distler, 2008 ; Berland et Essid, 2009), alors qu'ils sont pourtant au coeur des problématiques liées à la RG, notamment pour ce qui a trait à la mobilisation des parties prenantes internes.

La nécessité de disposer d'informations non financières pour mesurer et piloter la performance est un constat qui n'est pas récent dans le domaine du contrôle de gestion. Anthony (1972) l'évoquait déjà il y a près de 40 ans, et de nombreux outils comme le balanced scorecard ou le tableau de bord ont été développés depuis pour répondre à ce besoin. En revanche, le fait que la performance non financière puisse se rapporter à des éléments de RG constitue lui une nouveauté. Certains outils de pilotage proposés aujourd'hui dans la littérature en contrôle de gestion abordent la problématique de la mesure de la performance de la RG. En revanche, il existe, tout compte fait, peu de travaux empiriques récents relatifs aux pratiques effectives des entreprises.

Partant de ce constat, la recherche poursuit deux objectifs. Le premier consiste à évaluer dans quelle mesure les entreprises développent des indicateurs de RG dans leurs outils de pilotage du contrôle de gestion. Le second s'attache à rechercher les facteurs qui expliquent les pratiques observées. L'article se subdivise en deux parties. Il débute par une revue de la littérature qui permet de définir la problématique et les hypothèses de la recherche. La deuxième partie présente la méthode utilisée et analyse les résultats obtenus.

\section{Revue de la littérature et hypothèses de la recherche}

Plusieurs approches ont été développées ces dernières années pour intégrer la dimension de la RG dans les systèmes de contrôle des entreprises. La comptabilité environnementale qui vise à évaluer les coûts liés à la prise en compte par les entreprises de l'environnement constitue l'une d'entre elles (Antheaume, 2005). Le bilan sociétal qui est destiné à évaluer les actions en faveur de la responsabilité sociale des entreprises à l'égard des partenaires et de l'environnement en est une autre (Capron, 2003), et certains auteurs s'interrogent également sur le rôle que pourraient jouer les principes et règles comptables dans l'amélioration de la qualité du reporting sociétal (Quairel, 2004). 
Parce qu'il appartient au contrôle de gestion d'organiser la performance de l'entreprise, d'en identifier les origines et d'en effectuer la mesure (Bouquin, 1994), et dès lors que le concept de RG est de plus en plus présent dans la stratégie des entreprises, il apparaît pertinent d'analyser également dans quelle mesure les systèmes de pilotage (ou de mesure) de la performance du contrôle de gestion intègrent les aspects environnementaux, sociaux et sociétaux de la performance. Cela doit permettre à la fois d'évaluer l'impact des stratégies de RG sur les outils de contrôle de gestion, et de rendre compte indirectement de l'effectivité de l'engagement des parties prenantes internes dans la mise en oeuvre des démarches qui y sont associées. Ces systèmes qui se déclinent sous différentes formes (tableau de bord, balanced scorecard, navigateur, pour les versions les plus répandues), ont pour fonction principale de: «traduire la mission et la stratégie de l'entreprise en un ensemble d'indicateurs de performance qui constituent la base d'un système de pilotage de la stratégie » (Kaplan et Norton, 1996).

\subsection{Les systèmes de mesure de la performance du contrôle de gestion et la RG}

Une revue de la littérature permet de constater qu'il existe des propositions quant au pilotage de la performance de la RG par les outils du contrôle de gestion.

Combinaison de mesures financières et non financières organisées selon quatre axes d'analyse: les résultats financiers, la satisfaction des clients, les processus internes, l'innovation et l'apprentissage organisationnel, le balanced scorecard ne faisait pas référence dans sa première version à la problématique de la RG (Kaplan et Norton, 1996). Cette question est aujourd'hui évoquée par ses concepteurs qui considèrent que la capacité de l'entreprise à être citoyenne doit faire partie intégrante de la mesure de la performance et qui suggèrent de développer des indicateurs sur l'axe " processus internes " et d'étendre l'axe " clients " à tous les partenaires de l'entreprise (Kaplan et Norton, 2001). En adoptant un cadre d'analyse relativement proche de celui de Kaplan et Norton, Gminder et Bieker (2002) suggèrent quant à eux d'ajouter un cinquième axe " sociétal " au balanced scorecard en étroite relation avec les quatre autres dimensions plus classiques. Il faut noter d'ailleurs que le balanced scorecard est souvent évoqué dans la littérature anglo-saxonne qui traite de la RG (Zingales et al. 2002).

Puisant ses fondements conceptuels dans la notion de capital intellectuel, le navigateur, théorisé par Edvinsson et Malone et mis en oeuvre chez Skandia AFS (Edvinsson et Malone, 1999) tire son originalité du fait qu'il se concentre sur l'une des dimensions de la RG. Le navigateur porte en effet une attention particulière aux ressources humaines qui sont positionnées au coeur du dispositif de création de valeur et pour lesquelles il existe en théorie autant d'indicateurs que pour les autres dimensions de la performance. Une partie de la performance sociale, celle liée aux salariés de l'entreprise, semble donc prise en compte par le navigateur. L'axe humain correspond aux compétences et attitudes des salariés et à l'engagement de l'entreprise de les maintenir à niveau.

D'autres approches du pilotage de la performance faisant référence à la problématique de la RG peuvent également être citées.

Le tableau de bord français enfin (Chiapello et Lebas, 2001), moins directif que les outils précédents dans son approche de la performance, ne recommande pas explicitement de développer des indicateurs liés à la RG. Néanmoins, sa démarche de construction articulée autour des étapes successives suivantes : définition des objectifs stratégiques, identification des variables d'action, choix des indicateurs, suppose implicitement que cette dimension peut être prise en compte dès lors que la RG est inscrite dans la stratégie de l'entreprise. Le pilotage de la performance de la RG est donc abordé par les outils du contrôle de gestion présentés dans la littérature. Or, certains travaux ont montré par le passé que sa mise en oeuvre demeurait peu répandue (Lingle et Schiemann, 1996 ; Ittner et al. 2003). II peut être intéressant de ce point de vue d'observer aujourd'hui les pratiques des entreprises et d'expliquer ce pourquoi celles-ci peuvent varier d'une organisation à l'autre. 


\subsection{Les hypothèses de la recherche}

Les travaux sur la contingence du contrôle de gestion ont montré que les caractéristiques des modes de contrôle en général et des systèmes de contrôle de gestion en particulier pouvaient varier d'une entreprise à l'autre sous l'effet de facteurs structurels. Une revue des résultats des principaux travaux empiriques qui se rapportent à cette approche (pour une synthèse de ces travaux, voir par exemple Chenhall (2003)) permet de poser des hypothèses quant à la manière dont les entreprises seraient susceptibles d'intégrer la mesure de la performance de la RG dans leurs outils de pilotage du contrôle de gestion. Six facteurs pouvant éventuellement expliquer les pratiques des entreprises ont été retenus. II s'agit de la taille de l'entreprise, de la stratégie, de l'incertitude perçue de l'environnement, de la nationalité de l'entreprise, de la cotation en bourse, du secteur d'activités.

\subsubsection{La taille}

Plusieurs recherches ont montré que la taille avait une influence positive sur la sophistication des systèmes de contrôle de gestion, notamment sur le degré de développement des indicateurs de performance non financière (Hoque et James, 2000 ; Moores and Yuen, 2001). Au regard de ce résultat et du fait, comme le note Damak-Ayadi (2006) que les grandes entreprises subissent des pressions plus importantes de la part de leurs " parties prenantes ", on peut s'attendre à ce que le pilotage de la performance en matière de RG soit plus développé dans les entreprises de grande taille. L'hypothèse suivante est donc posée :

$H 1$ : La présence des indicateurs de performance relatifs à la $R G$ dans les outils de pilotage du contrôle de gestion est positivement reliée à la taille des entreprises.

\subsubsection{La stratégie}

Le lien entre la stratégie et les systèmes de contrôle de gestion repose sur l'idée que l'importance plus ou moins grande accordée par l'entreprise à différents aspects de sa performance dépend étroitement de son orientation stratégique.

Les entreprises qui s'orientent vers des stratégies de domination pour les coûts privilégient des systèmes de contrôle de gestion centrés sur les coûts et les informations financières. A l'inverse, les indicateurs non financiers sont plus présents dans les entreprises qui adoptent des stratégies de différenciation (Shank et Govindarajan, 1993). Or, les entreprises qui optent pour ces stratégies sont vraisemblablement plus sensibles aux influences externes, aux besoins des consommateurs et aux tendances de la société en général. Ceci conduit à poser l'hypothèse suivante :

H2 : Les indicateurs de performance relatifs à la RG sont plus présents dans les outils de pilotage du contrôle de gestion des entreprises qui adoptent une stratégie de différenciation

\subsubsection{L'incertitude perçue de l'environnement}

Parmi les recherches qui ont analysé la relation entre l'incertitude de l'environnement et les systèmes de contrôle de gestion, certaines se sont particulièrement intéressées à l'influence de ce facteur de contingence sur le contenu des outils de contrôle de gestion. Chenhall et Morris (1986), Chia (1995) constatent ainsi que l'augmentation de l'incertitude perçue de l'environnement entraîne un recours plus important aux informations externes et non financières. Gosselin et Dubé (2002) montrent que les entreprises " prospectrices " qui doivent faire face à un niveau élevé d'incertitude contextuelle emploient davantage de mesures de performance non financières que les entreprises " défenderesses " qui évoluent dans un environnement plus stable. Bescos et al. (2004) observent que les managers sont plus critiques vis-à-vis des budgets qui sont centrés sur l'aspect financier de la performance lorsque l'incertitude perçue de l'environnement est élevée. Chong et Chong (1997) expliquent que les entreprises ont besoin d'informations non financières pour faire face à l'incertitude de leur environnement. Les entreprises qui doivent tenir compte des évolutions de leur environnement sont, semble-t-il, plus à même d'intégrer les problématiques de RG. Ce constat conduit à poser l'hypothèse suivante :

H3 : Les indicateurs de performance relatifs à la RG sont plus présents dans les outils de pilotage du contrôle de gestion des entreprises qui perçoivent leur environnement comme étant incertain. 


\subsubsection{La nationalité de l'entreprise}

II est reconnu que les entreprises européennes, et plus particulièrement françaises, visent une plus grande harmonie entre les dimensions économiques et sociales de leurs actions que les entreprises anglo-saxonnes dont le modèle de gouvernance " contractualiste " privilégie la création de valeur financière pour l'actionnaire en postulant que la seule responsabilité d'une firme est d'accroître ses profits. Une étude de Ittner et al. (2003) montre d'ailleurs que les variables de performance relatives aux partenaires, aux fournisseurs, à la collectivité, à l'environnement sont considérées par les managers des entreprises nord américaines comme étant beaucoup moins importantes que celles liées aux clients, aux résultats financiers, à la qualité et aux opérations. L'hypothèse suivante est donc posée :

H4 : Les indicateurs de performance relatifs à la RG sont plus présents dans les outils de pilotage du contrôle de gestion des entreprises françaises que dans ceux des entreprises anglo-saxonnes.

\subsubsection{La cotation en bourse}

A l'exception de la recherche de Bescos et al. (2004), l'effet de la cotation en bourse sur les systèmes de contrôle de gestion a été très peu approfondi empiriquement. D'une manière générale, comme le rappellent les auteurs cités ci-dessus, les contraintes de communication de données financières sont plus fortes dans les entreprises cotées en bourse qui doivent rassurer leurs actionnaires et informer les marchés financiers de leur performance. On pourrait donc s'attendre à ce que ces entreprises se concentrent sur le seul aspect financier de leur performance. II semble néanmoins que les actionnaires sont de plus en plus sensibles aux problématiques de responsabilité sociale, d'éthique ou de développement durable. Comme le fait remarquer Pérez (2002), l'actionnaire " socialement responsable " est devenu un acteur conséquent et reconnu comme tel dans le monde de l'épargne et des investissements financiers. Pour illustrer son propos l'auteur rappelle que ce sont certains grands fonds de pension comme Calpers qui ont été à l'origine des principes de " corporate governance ", qui ont progressivement fait évoluer les comportements boursiers vers une plus grande prise en considération des exigences en matière de RG. L'hypothèse suivante est donc posée : $H 5$ : Les indicateurs de performance relatifs à la RG sont plus présents dans les outils de pilotage du contrôle de gestion des entreprises cotées en bourse.

\subsubsection{Le secteur d'activités}

Les entreprises du secteur industriel ont été, via la question du respect de l'environnement, les premières à être interpellées sur la problématique de la RG, parce que leurs activités présentaient un risque environnemental beaucoup plus important que celles des entreprises commerciales ou du secteur des services. D'ailleurs, la « Global Reporting Initiative » (GRI) qui rassemble des entreprises, des ONG, des organismes de comptabilité, des chercheurs et qui a pour mission de développer, au niveau international, des directives pour aider les entreprises à évaluer leurs performances économique, environnementale, et sociale, est une initiative qui s'est développée en 1997 à destination, dans un premier temps, des entreprises industrielles. On peut donc s'attendre à ce que les entreprises industrielles soient plus concernées par le pilotage de la RG. L'hypothèse suivante est donc posée :

$H 6$ : Les indicateurs de performance relatifs à la RG sont plus présents dans les outils de pilotage du contrôle de gestion des entreprises industrielles que dans ceux des autres entreprises.

\section{Méthode et résultats}

Pour observer les pratiques des entreprises et tester les hypothèses de la recherche, une étude empirique a été réalisée auprès d'un échantillon de grandes entreprises implantées en France.

\subsection{Le recueil des données}

Les données empiriques nécessaires à la recherche ont été collectées grâce à l'envoi d'un questionnaire par voie postale. L'étude a été réalisée, en janvier 2007, auprès d'un échantillon de 
grandes entreprises implantées en France. L'enquête quantitative a été choisie pour recueillir une quantité importante d'observations exploitables statistiquement. Le questionnaire a tout d'abord été testé auprès d'experts en contrôle de gestion (10 enseignants en contrôle de gestion et 10 contrôleurs de gestion). Il a été administré ensuite par voie postale à 400 contrôleurs de gestion évoluant dans des entreprises de 500 à 5000 salariés, sélectionnées de façon aléatoire dans la base de données Kompass. Une relance téléphonique a été effectuée 3 mois après l'envoi du questionnaire. 83 questionnaires ont finalement été retournés (taux de réponse de 20,75\%). 79 d'entre eux se sont avérés exploitables (voir tableau 1). Les entreprises n'ayant pas répondu au questionnaire ne sont pas significativement différentes de celles de l'échantillon de l'étude.

Tableau 1. Les principales caractéristiques des entreprises composant l'échantillon de l'étude

\begin{tabular}{|c|c|}
\hline Composition & $\mathrm{N}$ \\
\hline - Entreprises françaises & 58 \\
- Entreprises anglo-saxonnes & 15 \\
\hline - Entreprises cotées en bourse & 38 \\
- Entreprises non cotées en bourse & 41 \\
\hline - Entreprises industrielles & 45 \\
- Entreprises commerciales & 14 \\
- Entreprises de services & 20 \\
\end{tabular}

\subsection{La mesure des variables}

Pour observer le contenu des outils de pilotage du contrôle de gestion, il est demandé aux répondants d'évaluer sur une échelle de Likert à 5 points allant de 1 (très faible) à 5 (très élevé), le degré de présence des indicateurs financiers et des indicateurs non financiers relatifs aux variables clés de gestion (coûts, qualité, productivité, délai, etc...), au marché (satisfaction client, part de marché, etc...), aux éléments immatériels (ressources humaines, systèmes d'information, innovation, etc...), et enfin plus spécifiquement à la RG. De nombreux exemples, pour chacune des catégories d'indicateurs, sont fournis dans le questionnaire pour accroître la lisibilité de la question.

De façon complémentaire les enquêtés ont évalué également sur une échelle de Likert à 5 points allant de 1 (très faiblement) à 5 (très fortement), la mesure dans laquelle les cinq types d'indicateurs sont reliés aux objectifs stratégiques de l'entreprise.

L'incertitude perçue de l'environnement est évaluée à travers sept items provenant de l'instrument de mesure élaboré par Govindarajan (1984). Il est demandé aux répondants d'indiquer sur une échelle de Likert à 5 points allant de 1 (très prévisible(s)) à 5 (très imprévisible(s)), dans quelle mesure les éléments suivants sont difficiles à prévoir : la réglementation, les actions syndicales, la demande, les actions des concurrents, le comportement des clients, l'évolution des technologies, la disponibilité des matières premières ou des achats. Le score global d'incertitude perçue de l'environnement est évalué en calculant le score global obtenu sur les sept items.

L'instrument de mesure développé par Chenhall et Langfield-Smith (1998) a été choisi pour caractériser les stratégies adoptées par les entreprises. Les enquêtés ont ainsi exprimé sur une échelle de Likert à 5 points leur degré d'accord avec dix items représentatifs de dix propositions d'orientations stratégiques. Une analyse factorielle a été réalisée pour qualifier les stratégies des entreprises de l'échantillon. Trois axes factoriels ont été retenus pour résumer l'information de départ provenant des dix items. Les trois axes factoriels détiennent des valeurs propres de 3,$23 ; 1,81$ et 1,1 et représentent respectivement $32,28 \% ; 18,12 \%$ et $10,22 \%$ de la variance du nuage de points initial (soit $60,62 \%$ au total). La première composante principale relie des items liés à la 
qualité des produits et des services, à leur disponibilité, et au respect des délais de livraison. La seconde qualifie les stratégies de domination par les coûts. La troisième composante principale indique une corrélation significative des items représentatifs des stratégies centrées sur l'innovation. Les trois stratégies mises en évidence sont similaires à celles observées par les recherches antérieures qui ont utilisé le même instrument d'observation (Bescos et al. 2004).

Il a été demandé aux répondants d'indiquer enfin la taille et la nationalité de l'entreprise, ainsi que son secteur d'activité.

\subsection{Les résultats sur la présence des indicateurs de RG}

Globalement les premiers résultats de la recherche montrent que les indicateurs de performance liés à la RG sont en moyenne relativement peu présents dans les outils de pilotage du contrôle de gestion des entreprises de l'échantillon. Ils sont notamment beaucoup moins représentés en moyenne que les indicateurs des autres dimensions plus classiques de la performance (tableau 2).

Tableau 2- La moyenne du niveau de présence des indicateurs classés par catégorie dans les outils de pilotage du contrôle de gestion

\begin{tabular}{|l|c|c|c|c|l|}
\hline \multicolumn{1}{|c|}{ Type d'indicateurs } & Financier & Marché & $\begin{array}{l}\text { Variables } \\
\text { clés de } \\
\text { gestion }\end{array}$ & Immatériel & RG \\
\hline $\begin{array}{l}\text { Niveau de présence } \\
\text { des indicateurs } \\
\text { (moyenne sur 5 points) }\end{array}$ & 4,25 & 2,75 & 2,85 & 2,4 & 1,65 \\
\hline
\end{tabular}

Les pratiques des entreprises sont néanmoins différenciées puisque trois des six hypothèses sont validées (figure 1). Les indicateurs de performance liés à la RG sont plus présents dans les outils de pilotage du contrôle de gestion des organisations de grande taille (validation de $\mathrm{H} 1$ ) et dans les entreprises cotées en bourse (validation de H5). Les entreprises industrielles y recourent également plus que les entreprises commerciales (validation partielle de $\mathrm{H} 6$ ). II existe par ailleurs des écarts entre les pratiques des entreprises françaises et anglo-saxonnes et entre celles des entreprises industrielles et des entreprises du secteur des services, mais les différences ne sont pas significatives. Enfin, les corrélations entre les variables représentatives des types de stratégie, celle caractéristique de l'incertitude perçue de l'environnement et le niveau de présence des indicateurs de RG dans les outils de pilotage du contrôle de gestion ne sont pas significatives statistiquement (Tous ces résultats sont présentés en détail dans les tableaux 3 et 4 ).

Figure 1. L'influence des facteurs de contingence sur la présence des indicateurs de RG dans les outils de pilotage du contrôle de gestion

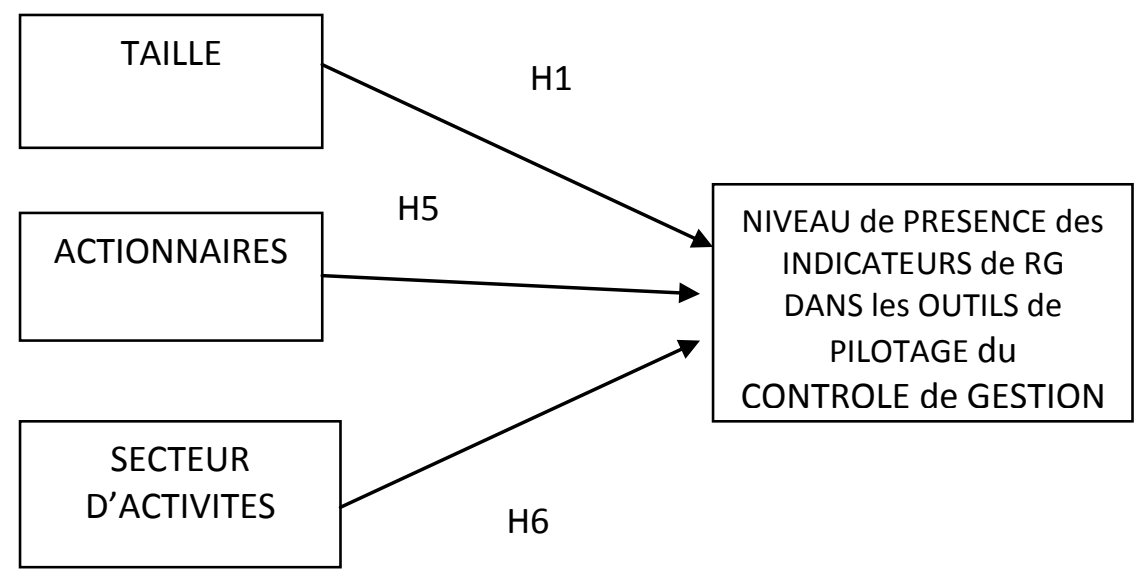


Tableau 3- Les résultats des tests de comparaison de la moyenne du niveau de présence des indicateurs de RG par facteurs de contingence

\begin{tabular}{|c|c|c|c|c|}
\hline & $\mathrm{N}$ & $\begin{array}{l}\text { Niveau de présence des indicateurs de } \\
\text { RG (moyenne évaluée sur } 5 \text { points) }\end{array}$ & Ecarttype & Ecart \\
\hline $\begin{array}{l}\text { - Entreprises françaises } \\
\text { - Entreprises anglo-saxonnes }\end{array}$ & $\begin{array}{l}58 \\
15\end{array}$ & $\begin{array}{c}1,67 \\
2\end{array}$ & $\begin{array}{l}1,02 \\
1,14\end{array}$ & $0,33 n s$ \\
\hline $\begin{array}{l}\text { - Entreprises cotées en bourse } \\
\text { - Entreprises non cotées en } \\
\text { bourse }\end{array}$ & $\begin{array}{l}38 \\
41\end{array}$ & $\begin{array}{l}1,92 \\
1,61\end{array}$ & $\begin{array}{l}1,17 \\
0,91\end{array}$ & $0,31 *$ \\
\hline $\begin{array}{l}\text { - Entreprises industrielles } \\
\text { - Entreprises commerciales }\end{array}$ & $\begin{array}{l}45 \\
14\end{array}$ & $\begin{array}{l}1,93 \\
1,50\end{array}$ & $\begin{array}{l}1,05 \\
0,76\end{array}$ & $0,43^{*}$ \\
\hline $\begin{array}{l}\text { - Entreprises industrielles } \\
\text { - Entreprises de services }\end{array}$ & $\begin{array}{l}45 \\
20\end{array}$ & $\begin{array}{l}1,93 \\
1,55\end{array}$ & $\begin{array}{l}1,05 \\
1,19\end{array}$ & $0,38 n s$ \\
\hline
\end{tabular}

* écart significatif au seuil de 0,05; ns écart non significatif.

Tableau 4 - Les résultats des tests de corrélation entre la taille, l'incertitude perçue de l'environnement, les types de stratégie et le niveau de présence des indicateurs de RG dans les outils de pilotage du contrôle de gestion.

\begin{tabular}{|l|l|}
\hline & Niveau de présence des indicateurs de RG \\
\hline Taille & $\mathbf{0 , 3 3 *}$ \\
\hline Stratégie orientée qualité et délai & NS \\
\hline Stratégie de domination par les coûts & NS \\
\hline Stratégie d'innovation & NS \\
\hline Incertitude perçue de l'environnement & NS \\
\hline
\end{tabular}

* corrélation significative au seuil de 0,01, NS corrélation non significative.

\subsection{Les résultats sur le degré de relation des indicateurs de RG avec les objectifs stratégiques}

Les indicateurs de RG sont, de tous les indicateurs, ceux qui sont le moins reliés aux objectifs stratégiques des entreprises (tableau 5). II faut noter néanmoins que le degré de relation croît lorsque l'entreprise est de grande taille (tableau 6), qu'elle évolue dans le secteur industriel ou lorsqu'elle est cotée en Bourse (tableau 7). 
Tableau 5- La moyenne du degré de relation des indicateurs de pilotage classés par catégorie avec les objectifs stratégiques de l'entreprise

\begin{tabular}{|l|l|l|l|l|l|}
\hline Type d'indicateurs & Financier & Marché & $\begin{array}{l}\text { Variables clés } \\
\text { de } \\
\text { gestion }\end{array}$ & Immatériel & RG \\
\hline $\begin{array}{l}\text { Degré de relation } \\
\text { avec } \\
\text { la stratégie } \\
\text { (moyenne } \\
\text { sur } 5 \text { points) }\end{array}$ & 4 & 3,68 & 3,06 & 2,95 & 2,22 \\
\hline
\end{tabular}

Tableau 6 - Les résultats des tests de corrélation entre la taille, l'incertitude perçue de l'environnement, les types de stratégie et le degré de relation des indicateurs de RG avec les objectifs stratégiques

\begin{tabular}{|c|l|}
\hline & $\begin{array}{l}\text { Degré de relation des indicateurs de RG avec la } \\
\text { stratégie }\end{array}$ \\
\hline Taille & $\mathbf{0 , 3 2}$ \\
\hline Stratégie orientée qualité et délai & NS \\
\hline Stratégie de domination par les coûts & NS \\
\hline Stratégie d'innovation & NS \\
\hline Incertitude perçue de l'environnement & NS \\
\hline
\end{tabular}

* corrélation significative au seuil de 0,01, NS corrélation non significative,

Tableau 7- Les résultats des tests de comparaison de la moyenne du degré de relation des indicateurs de $R G$ avec les objectifs stratégiques

\begin{tabular}{|c|c|c|c|c|}
\hline & $\mathrm{N}$ & $\begin{array}{l}\text { Degré de relation } \\
\text { des } \\
\text { indicateurs de RG } \\
\text { avec la } \\
\text { stratégie (moyenne } \\
\text { évaluée } \\
\text { sur } 5 \text { points) }\end{array}$ & Ecarttype & Ecart \\
\hline $\begin{array}{l}\text { - Entreprises françaises } \\
\text { - Entreprises anglo-saxonnes }\end{array}$ & $\begin{array}{l}58 \\
15\end{array}$ & $\begin{array}{l}2,12 \\
2,29\end{array}$ & $\begin{array}{l}1,10 \\
1,11\end{array}$ & $0,17 \mathrm{~ns}$ \\
\hline $\begin{array}{l}\text { - Entreprises cotées en bourse } \\
\text { - Entreprises non cotées en } \\
\text { bourse }\end{array}$ & $\begin{array}{l}38 \\
41\end{array}$ & $\begin{array}{c}2,39 \\
2\end{array}$ & $\begin{array}{c}1,30 \\
1\end{array}$ & $0,39 *$ \\
\hline $\begin{array}{l}\text { - Entreprises industrielles } \\
\text { - Entreprises commerciales }\end{array}$ & $\begin{array}{l}45 \\
14\end{array}$ & $\begin{array}{c}2,40 \\
2\end{array}$ & $\begin{array}{l}1,16 \\
1,23\end{array}$ & $0,40 *$ \\
\hline $\begin{array}{l}\text { - Entreprises industrielles } \\
\text { - Entreprises de services }\end{array}$ & $\begin{array}{l}45 \\
20\end{array}$ & $\begin{array}{l}2,40 \\
1,95\end{array}$ & $\begin{array}{l}1,23 \\
1,27\end{array}$ & $0,45 \mathrm{~ns}$ \\
\hline
\end{tabular}

* écart significatif au seuil de 0,01; ns écart non significatif. 


\subsection{L'analyse des résultats}

La recherche délivre deux enseignements importants. Le premier est que la mesure de la performance liée à la RG demeure relativement peu répandue dans les outils de pilotage du contrôle de gestion. Lorsque cette performance est suivie, alors les entreprises ne relient pas (ou très peu) les indicateurs qui la mesure à leurs objectifs stratégiques. D'ailleurs, il n'existe pas de lien entre le type de stratégie adoptée par les entreprises et le degré de développement des indicateurs de RG. Plusieurs explications peuvent être avancées pour analyser ces résultats.

La première consiste à penser que la RG peut être présente dans la stratégie des entreprises, mais que ces dernières attachent, tout compte fait, peu d'importance à l'effectivité de sa mise en oeuvre. Dans ce cas cela signifierait que les entreprises portent avant tout de l'attention à la RG à des fins de communication externe, sans que cela ne constitue une variable de performance pilotée en interne, et sans que les parties prenantes internes ne soient véritablement engagés dans les démarches qui en découlent. La recherche confirmerait alors empiriquement les propos de Naro (2005) qui mentionne que le lien entre les indicateurs sociétaux et la stratégie n'est très souvent pas effectif dans les entreprises et qu'il est donc légitime de penser que le contrôle de gestion dit " sociétal " se réduit aujourd'hui à de la communication à l'intention de différents publics externes à l'entreprise.

Le résultat peut également s'expliquer par le fait que les entreprises peuvent effectivement avoir développé des stratégies intégrant des dimensions de RG sans que cela ne se soit traduit par une évolution des outils de pilotage du contrôle de gestion au moment de l'enquête. Les données ont été collectées en 2007. Il se peut que les entreprises aient depuis fait évoluer leurs dispositifs en accord avec leur stratégie.

Il se peut enfin, qu'une partie du pilotage de la RG soit réalisée en dehors du périmètre du contrôle de gestion, par chaque service ou département ou bien par les Directions dédiées à la RG lorsque celles-ci existent au sein des entreprises (Mocquet, 2005). Si tel est le cas comme le montre certains travaux (Marquet- Pondeville et al. 2007), cela pose inévitablement la question de l'intégration des dispositifs de pilotage de la performance. Certains auteurs sont partisans d'un découplage des indicateurs de RG des outils de pilotage du contrôle de gestion (Quairel, 2006) au motif que les démarches associées aux premiers sont incompatibles avec celles attachées aux seconds, là où d'autres plaident en faveur d'une intégration dans un souci de cohérence (Bollecker et al. 2006 ; Meyssonier et Rasolofo - Distler, 2008 ; Berland et Essid, 2009). Bien que sa mise en oeuvre soit plus aisée, le premier schéma comporte un risque. Déconnectées de la stratégie et des objectifs économiques qui en découlent, les initiatives relatives à la responsabilité globale peuvent en effet être reléguées au rang de préoccupations secondaires. II remet par ailleurs en cause l'identité et le territoire du contrôle de gestion (Bouquin, 1993 ; Teller, 1999), sachant qu'il est reconnu pourtant depuis longtemps qu'il n'est plus pertinent de confiner les contrôleurs de gestion dans un rôle de " compteurs de haricots » (Drucker, 1990).

Globalement, et bien qu'elle soit minime par rapport au comportement d'ensemble des entreprises, une tendance doit néanmoins être signalée. L'effet des pressions et des contraintes externes liées à la taille, au secteur d'activité, et au fait que l'entreprise soit cotée en bourse joue un rôle positif significatif dans le développement des indicateurs de RG dans les outils de pilotage du contrôle de gestion. Le résultat concernant l'influence des actionnaires est notamment particulièrement intéressant. II montre qu'après avoir contribué à "financiariser " de façon parfois excessive la mesure de la performance des entreprises (Perez, 2002), les actionnaires sont aujourd'hui à l'origine de l'ouverture des outils de pilotage de la performance à des variables non financières relevant de la RG.

\section{Conclusion}

La recherche avait pour objectifs d'observer la place que les indicateurs de performance liés à la RG occupent dans les outils de pilotage de contrôle de gestion des entreprises et d'identifier certains facteurs pouvant être à l'origine des pratiques. Les résultats d'une enquête réalisée en janvier 2007 auprès de 79 grandes entreprises implantées en France montrent que les systèmes de mesure de la performance du contrôle de gestion intègrent en moyenne relativement peu d'indicateurs liés à la 
RG. Ces indicateurs, lorsqu'ils sont présents, sont en moyenne peu reliés aux objectifs stratégiques. Les pratiques des entreprises sont néanmoins différenciées. Les organisations de grande taille, les entreprises industrielles sensibles au risque environnemental et les entreprises cotées en Bourse soumises à la pression d'actionnaires de plus en plus soucieux des questions relevant de la RG ont tendance à développer, plus que les autres entreprises, un pilotage de la RG au sein de leurs dispositifs de contrôle de gestion. En revanche, le type de stratégie, l'incertitude perçue de l'environnement et la nationalité de l'entreprise n'ont pas d'influence sur le niveau de développement des indicateurs de RG dans les outils de pilotage du contrôle de gestion.

La recherche comporte bien entendu des limites. Compte tenu du caractère déclaratif des données collectées, il se peut qu'il existe un écart entre les discours et la réalité concernant le niveau de présence des indicateurs de RG dans les outils de pilotage du contrôle de gestion. Elle suggère également des développements ultérieurs. Les résultats obtenus peuvent être approfondis par une analyse des rapports annuels des entreprises de l'échantillon qui sont cotées. Ils peuvent également être analysés sous un angle théorique différent en mobilisant le cadre des théories néoinstitutionnelles qui postulent que le comportement des entreprises tend à s'homogénéiser sous l'effet des pressions institutionnelles. L'hypothèse que les pratiques des entreprises résultent d'attitudes mimétiques (Quairel, 2005), ou de la nécessité de se conformer à des règlementations, à des normes (Evraert, 2000 ; Mikol, 2000 ; Mocquet et Pezet, 2006) peut en effet être étudiée. Enfin, il serait intéressant d'analyser dans quelle mesure les facteurs subjectifs liés aux acteurs influencent les pratiques observées. Toutes ces pistes demandent à être explorées pour prolonger les résultats de cette recherche. 


\section{Bibliographie}

Antheaume N. (2005), "La comptabilité environnementale est-elle une forme d'hypocrisie organisationnelle ? Le cas des indicateurs de performances écologiques d'une gamme de pesticides ", Congrès de l'Association Francophone de Comptabilité, Lille.

Anthony R.N. (1972), "Accounting for the Future ", Sloan Management Review, Vol. 13, spring, p. 17-34.

Berland N. et Essid M. (2009), "RSE, système de contrôle et pilotage de la performance globale ", Congrès de l'Association Francophone de Comptabilité, Strasbourg.

Bescos P.L., Cauvin E., Langevin P. et Mendoza C. (2004), " Critiques du budget : une approche contingente ", Revue Comptabilité, Contrôle, Audit, Vol. 10, No 1, p. 165-185.

Bollecker M., Mathieu P. et C. Clementz (2006), « Le comportement socialement responsable des entreprises : une lecture des travaux en comptabilité et contrôle de gestion dans une perspective néo-institutionnaliste ", Congrès de l'Association Francophone de Comptabilité, Tunis.

Bouquin H. (1993), Comptabilité de gestion, Dalloz.

Bouquin H. (1994), Les fondements du contrôle de gestion, PUF.

Capron M. (2003), "Un nouvel instrument d'auto-évaluation des organisations : le bilan societal », Revue Comptabilité, Contrôle, Audit, Nº spécial, p. 55-70.

Chenhall R.H. (2003), " Management Control Systems Design within its Organizational Context; Findings from Contingency-based Research and Directions for the Future ", Accounting, Organizations and Society, Vol. 28, No 2-3, p. 127-163.

Chenhall R.H. \& Langfield-Smith K. (1998), " The Relationship between Strategic Priorities, Management Techniques and Management Accounting: an Empirical Investigation Using a System Approach ", Accounting, Organization and Society, Vol. 23, No 3, p. 243-264.

Chenhall, R.H. \& Morris D. (1986), " The Impact of Structure, Environment, and Interdependence on the Perceived Usefulness of Management Accounting Systems ", The Accounting Review, Vol. 66, N 1, p. 16-35.

Chia, Y.M. (1995), " Decentralization, Management Accounting Systems (MAS) Information Characteristics and Their Interaction Effects on Managerial Performance: a Singapore

Study ", Journal of Business Finance and Accounting, Vol. 22, N 6, p. 811-830.

Chiapello E. et Lebas, M. (2001), " The Tableau de Bord, French Approach to Management Information ", Cahier de recherche, HEC.

Chong, V.K. \& Chong K.M. (1997), " Strategic Choices, Environmental Uncertainty and SBU Performance: A Note on the Intervening Role of Management Accounting Systems ", Accounting and Business Research, Vol. 27, N 4, p. 268-276.

Damak-Ayadi S. (2006), « Analyse des facteurs explicatifs de la publication des rapports sociétaux en France ", Comptabilité, Contrôle, Audit, Tome 12, Vol. 2, p. 73-116. 
Drucker P.F. (1990), " The Emerging Theory of Manufacturing ", Harvard Business Review, Vol. 68, N 3, p. 94-102.

Edvinsson L. et M.S. Malone (1999), Le capital immatériel de l'entreprise, Paris, Maxima Laurent du Mesnil Editeur.

Evraert S. (2000), "Confiance et comptabilité », in Encyclopédie de comptabilité, contrôle de gestion et audit, Economica, p. 461-471.

Freeman R.E. (1984), Strategic Management: A Stakeholder Approach, Boston, Pitman.

Gminder C.U \& Bieker T. (2002), " Managing Corporate Sustainability by Using the SustainabilityBalanced Scorecard ", Contribution to the 10th International Conference of the Greening of Industry Network, Göteborg.

Gosselin M. et Dubé T. (2002), "Influence de la stratégie sur l'adoption des mesures de performance en vigueur dans le système de comptabilité de gestion ", Actes du congrès de l'Association Francophone de Comptabilité, Toulouse.

Govindarajan V. (1984), "Appropriateness of Accounting Data in Performance Evaluation: an Empirical Investigation of Environmental Uncertainty as an Intervening Variable», Accounting, Organization and Society, Vol. 9, N², p. 125-135.

Hoque Z. \& James W. (2000), " Linking Balanced Scorecard Measures to Size and Market Factors: Impact on Organizational Performance ", Journal of Management Accounting Research, Vol. 12, p. 117.

Ittner, C.D., Larcker D.F. \& Randall T. (2003), «Performance Implications of Strategic Performance Measurement in Financial Services Firms", Accounting, Organizations andSociety, Vol. 28, N 7/8, p. 715-741.

Kaplan R.S. \& Norton D.P. (1996), The Balanced Scorecard, The Harvard Business School Press.

Kaplan R.S. et Norton D.P. (2001), Comment utiliser le tableau de bord prospectif, Editions d'organisation.

Lingle J.H. \& W.A. Schiemann W.A. (1996), " From Balanced Scorecard to Strategic Gauges: Is Measurement Worth It? ", Management Review, Vol. 85, N³, p. 56-61.

Marquet-Pondeville S., Swaen V. et De Rongé Y. (2007), "The Impact of External Environment, Stakeholders Pressures and Environmental Strategy on Environmental Management Control Systems ", Congrès de l'Association Francophone de Comptabilité, Poitiers.

Martinet A. et Reynaud E. (2001), "Shareholders, stakeholders et stratégie ", Revue Française de Gestion, N 136 , p. 12-25.

Meyssonnier F. et Rasolofo-Distler F. (2008), " le contrôle de gestion entre responsabilité globale et performance économique : le cas d'une entreprise sociale pour l'habitat ", Revue Comptabilité Contrôle-Audit, Tome 14 - Vol. 2 - Décembre.

Mikol A. (2000), "L'information environnementale publiée par les sociétés du CAC 40 de 1992 à 1998 comparée à une information type ", Congrès de l'Association Française de Comptabilité, Angers. 
Mocquet A.C., (2005), « De l'intégration du développement durable dans la stratégie au pilotage et à l'instrumentation de la performance globale ", Revue Management et Avenir, N 3, p. 153-170.

Mocquet A.C. et Pezet A. (2005), "Les technologies de la responsabilité sociale ou l'invention du manager responsable : le cas Lafarge ", Revue Finance Contrôle Stratégie, Vol. 9, № 3, p. 113-142.

Moores K. \& Yuen S. (2001), « Management Accounting Systems and Organizational Configurations: a Life-Cycle Perspective », Accounting Organizations and Society, Vol. 26, № 4/5, p. 351-389.

Naro G. (2005), "Responsabilité sociale de l'entreprise et pilotage des performances », in Le Roy F. et Marchesnay M., La responsabilité sociale de l'entreprise, Editions EMS, p. 59-71.

Pérez R. (2002), "L'actionnaire socialement responsable : mythe d'hier ou réalité de demain ? ", Revue Française de Gestion, № 141, p. 131-151.

Quairel F. (2004), "Responsable mais pas comptable : analyse de la normalisation des rapports environnementaux et sociaux ", Revue Comptabilité, Contrôle, Audit, Tome 10, Vol. 1, p. 7-36.

Quairel F. (2005), " La mesure de la performance sociétale à l'aune de l'instrumentation comptable et financière ", in Le Roy F. et Marchesnay M., La responsabilité sociale de l'entreprise, Editions EMS, p. 73-87.

Quairel F. (2006), "Contrôle de la performance globale et RSE ", Actes du Congrès Annuel de l'Association Francophone de Comptabilité, Tunis.

Shank J.K. \& Govindarajan V. (1993) Strategic Cost Management, the New Tool for Competitive Advantage, New York, The Free Press.

Teller R. (1999), Le contrôle de gestion, pour un pilotage intégrant stratégie et finance, Editions Management et Société.

Zingales F., O’Rourke A. \& Hockerts K. (2002), « Balanced Scorecard and Sustainability, State of the Art Review ", Working Paper, CMER, Insead. 